\title{
BENCH MEASUREMENTS OF LOW FREQUENCY TRANSVERSE IMPEDANCE
}

\author{
A. Mostacci, Univ. di Roma "La Sapienza", Rome, Italy, \\ F. Caspers, L. Vos, CERN, Geneva, Switzerland, \\ U. Iriso, BNL, Upton, New York, 11973, USA.
}

\begin{abstract}
For frequencies below $10 \mathrm{MHz}$ the classical two wire transmission line method is subject to difficulties in sensitivity and measurement uncertainties. Thus for evaluation of the low frequency transverse impedance properties of the LHC dump kicker a modified version of the two wire transmission line has been used. It consists, in the present case, of a 10 turn loop of approximately 1 meter length and $2 \mathrm{~cm}$ width. The change of input impedance of the loop is measured as a function of the surroundings and by using a proper reference (metallic beam pipe) these changes are converted into a meaningful transverse beam coupling impedance. Measurements of several calibration objects have shown close agreement with theoretical results.
\end{abstract}

\section{INTRODUCTION}

A beam that oscillates from side to side with amplitude $\pm \Delta$ induces differential currents and charges on the walls of the vacuum chamber. These in turn produce a transverse magnetic field and an electric field which further deflects the beam. The threshold for beam instability and the growth rates depends on the so called transverse coupling impedance $Z_{T}$.

Transverse impedances are well known in literature and they can be theoretically calculated for a number of particularly simple structures or in general, they are numerically computed with codes. In this paper we are interested on bench measurements techniques, in particular at low frequencies (below few $\mathrm{kHz}$ ). At those frequencies a better sensitivity can be obtained by using a loop [1], instead of the classical two wire technique [2].

The interest for very low frequencies is directly related to the low transverse oscillation frequencies of future large machines (like LHC). To validate the bench measurement technique, measurements were carried out on a stainless steel pipe (with conductivity $\sigma=1.310^{6} \mathrm{~S} / \mathrm{m}$ ) for which theoretical results are available. The radius of the pipe is $b=50 \mathrm{~mm}$, its wall thickness is $t=1.5 \mathrm{~mm}$ and its length is $L=50 \mathrm{~cm}$. This paper reports those preliminary measurements and asseses a measurement recipe to be applied later to the more complex geometries of LHC devices.

\section{TRANSVERSE IMPEDANCE BENCH MEASUREMENTS}

Being $I$ the beam current, the source of the differential wall current is the dipole $I \Delta$ per unit length of the beam.
The same wall currents and magnetic (deflecting) field result if the beam is replaced by two parallel wires or more simply by a loop of length $L$, width $\Delta$ and current $I$. The magnetic field induces a voltage in the loop which increases its impedance (the current $I$ is constant). This additional impedance is simply the variation of the loop impedance when inserted in the Device Under Test (DUT) with respect to the loop impedance. Assuming that the loop is coiled $N$ times, the transverse coupling impedance can be obtained from

$$
Z_{T}=\frac{c}{\omega} \frac{Z^{D U T}-Z^{f . s .}}{N^{2} \Delta^{2}}
$$

where $Z^{D U T}$ is the (measured) impedance of the loop when inserted in the DUT and $Z^{f . s}$. is the (measured) impedance of the loop far away from any perturbing object (i.e. in free space).

In general, when measuring a very small impedance (as pointed out in [1]), one should also subtract the radiation resistance from the loop measurements in free space. This is appreciable unless the loop is very short compared with the wavelength. Alternatively one could place the loop in a circular perfectly conducting pipe (a copper or brass one is enough), for which the added impedance is easy to calculate.

\section{MEASUREMENT SET-UP}

The coil used in the measurement was $L_{w}=1.25 \mathrm{~m}$ long and $\Delta=22.5 \mathrm{~mm}$ wide. To reduce the signal to noise ratio (particularly important in our case since the measured signals are very small), the loop was coiled $N=10$ times. In this way, one can increase the useful signal with a factor $N^{2}$ with a drawback of lowering the frequency of loop self resonances: the chosen number of turns is a compromise to keep the lowest self resonance above $1 \mathrm{MHz}$.

The loop input impedance (much lower than $50 \mathrm{Ohm}$ ) was measured with an Agilent Vector Network Analyser (VNA4395A) using two different S-parameters test sets, according to the two different frequency ranges of the measurement. In the higher frequency range $(10 \mathrm{kHz}-1 \mathrm{MHz})$, a standard transformer type directional coupler (provided with the instrument as an impedance measurement test set) could be safely used. For lower frequencies $(100 \mathrm{~Hz}-$ $10 \mathrm{kHz}$ ), a resistive coupling network has been used: the $\mathrm{RF}$ output signal from the instrument is split and partly used for reference (the A signal in Fig. 1) and partly directed to the measurement coil (the DUT in Fig. 1). The reflected signal $(\mathbf{B})$ is then measured and a $20 \mathrm{db}$ attenuator 
(corresponding to dashed white line in Fig. 1) prevents the signal from the DUT from affecting the reference.

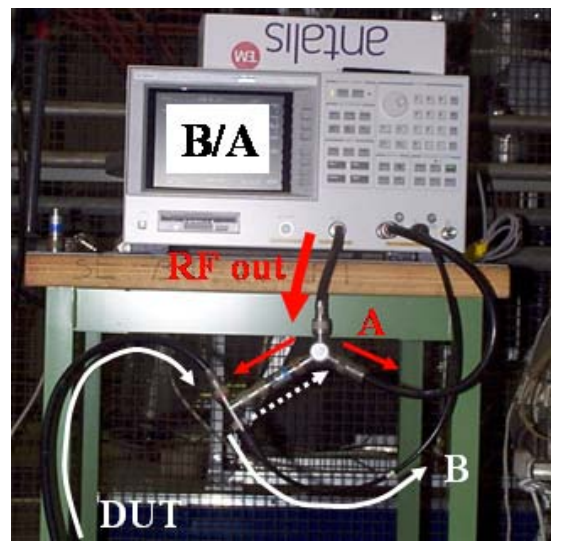

Figure 1: Set up for the low frequency measurements.

\section{CIRCULAR STEEL TUBE}

The transverse impedance of a circular pipe is well known and closed formulae are available, at least in the most significant limits. In the following we report the ones used to compare to the experimental data.

Being $\delta=\sqrt{2 /(\omega \mu \sigma)}$ the skin depth, $L$ the length of the structure (which in our case is $L=50 \mathrm{~cm}$ ), the transverse impedance for a circular beam pipe is for the "thick wall" case (see for example [3])

$$
\frac{Z_{T}}{L}=\frac{1+j}{\pi} \frac{c}{\omega \sigma b^{3} \delta} \quad \text { for } \quad \delta \ll t,
$$

where $c$ is the speed of light. For wall thickness smaller than the skin depth, a better approximation for the real transverse impedance can be obtained by using the wall thickness $t$ instead of $\delta$ in Eq. (2); anyway both formulae are not suited for very low frequencies where they diverge.

At low frequencies such that the skin depth is greater than the wall thickness, the interaction between the loop and a cylindrical resistive pipe can be described with an equivalent circuit model and the resulting transverse impedance is

$$
\frac{Z_{T}}{L}=\frac{c}{\pi b^{3} \sigma \omega t \Delta^{2}} \frac{1+j \omega_{c} / \omega}{1+\left(\omega_{c} / \omega\right)^{2}} \quad \text { with } \quad \omega_{c}=\frac{2}{\pi \mu b \sigma t}
$$

which is a corrected version of the result in Ref. [1].

An interesting derivation of the transverse impedance of a circular pipe with arbitrary surface impedance has been recently published in [4].

A field matching approach has been proposed to describe the impedance of a vacuum chamber with wall thickness smaller than the skin depth, in the framework of the VLHC studies. The results for a circular beam pipe are reported in Ref. [5] and the interested reader can find the closed analytical formulae in Ref. [6].
The measurement results on the steel pipe are compared to the theoretical expectation in Fig. 2. The thick wall formula $(\delta \ll t)$ of Eq. ( 2$)$ is a reasonable approximation only above $85 \mathrm{kHz}$ (light blue line); a good agreement over the whole range is achieved with an analytical model (brown line) and with the field matching of Ref. [6] (green diamonds). At low frequencies also the equivalent circuit model of Eq. (3) gives reasonable results (red line). In conclusion all the theoretical expectations for the real transverse impedance agree in their different validity ranges and the measurement (dark blue line) is coherent with them.

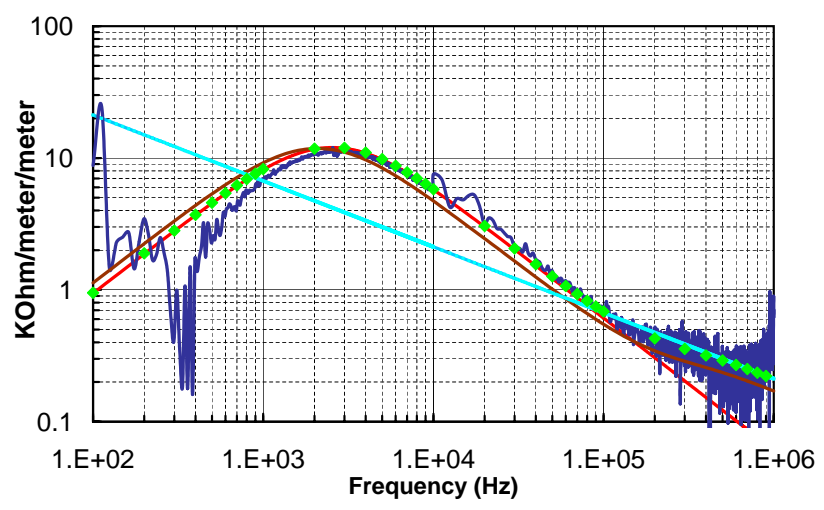

Figure 2: Transverse impedance per unit length (real part) of a steel circular pipe.

On the contrary for the imaginary part, the theoretical expectation are all in agreement, but they substantially differ from the measured values. In particular the measurements give a negative the immaginary part, while the theories predicts positive values. The results are shown in Fig. 3, with the same notation of Fig. 2.

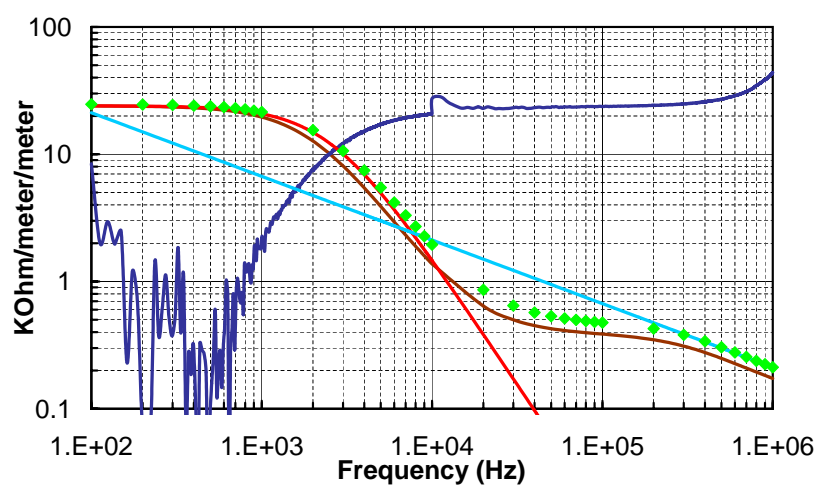

Figure 3: Transverse impedance per unit length (imaginary part, absolute value) of a steel circular pipe.

\section{REFERENCE MEASUREMENT ON A BRASS PIPE}

From the measurement shown above there is a clear contradiction between the expected imaginary transverse impedance (which saturates for very low frequencies) and 
the measured one (which vanishes at low frequencies). This controversy is important to solve since a consistent measurement recipe is needed for transverse impedance measurements on real LHC devices. For the moment the transverse impedance is obtained from the added loop impedance on the coil in the the DUT compared to a reference (lossless) case, i.e. the free space.

The inductance of two wires (at a distance $\Delta$ ) is different if they are placed in the free space or inside a cylindrical conductor of radius $b$. The difference between the inductances in the two cases is independent from the conductor properties (in particular from its conductivity) and it is proportional to $(\Delta / b)^{2}$. Therefore one can show $[1,4]$ that, according to Eq. (1), the (imaginary) transverse impedance of a perfectly conducting pipe (of radius $b$ ) is different from zero and equal to the "magnetic image transverse impedance" [4]

$$
Z_{T}^{p . c .}=-j \frac{Z_{0}}{2 \pi b^{2}} .
$$

For the brass pipe used in measurement (of the same geometry of the steel pipe, i.e. $b=50 \mathrm{~mm})$, one gets $Z_{T}^{p . c .}=$ $-j 24 \mathrm{k} \Omega$. The measurements shown in Fig. 4 agree (within the measurement uncertainties) with that prediction: the real part is mostly zero while the imaginary part is about $-23 \mathrm{k} \Omega$ in the plateau. The low frequency deviations are due the measurement difficulties while at high frequency (above $200 \mathrm{kHz}$ ) the effect of the loop self resonance becomes not negligible.

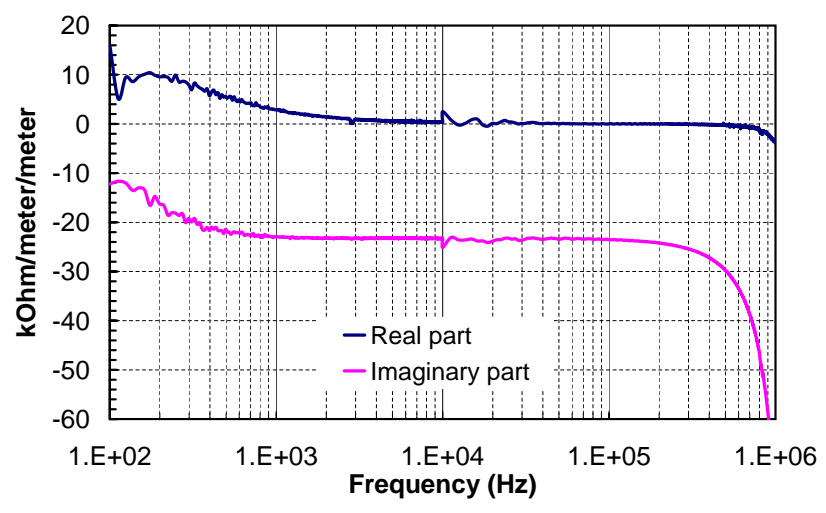

Figure 4: Transverse impedance per unit length of a brass circular pipe, according to Eq. (1).

In conclusion, to properly measure the transverse impedance, one should compute the added loop impedance with respect to the perfectly conducting pipe, i.e.

$$
Z_{T}=\frac{c}{\omega} \frac{Z^{D U T}-Z^{p . c .}}{N^{2} \Delta^{2}}
$$

must be used instead of Eq. (1). $Z^{p . c .}$ is the impedance of the loop inside a perfectly conducting pipe with the same geometry of the DUT.

By applying Eq. (5) to the steel cylindrical pipe, the real transverse impedance is not substantially modified, while the imaginary part saturates for low frequencies (as one should expect from theory). Figure 5 shows the measurement results, with the same notation of Fig. 2.

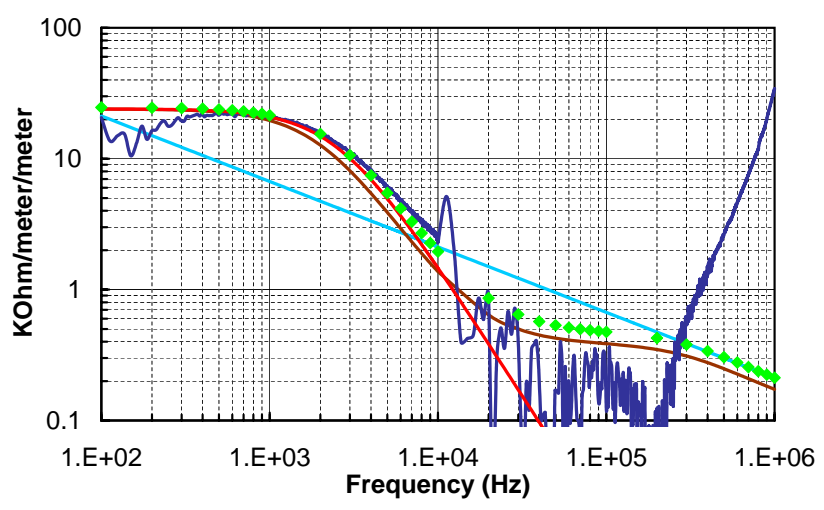

Figure 5: Transverse impedance per unit length (imaginary part) of a steel circular pipe after comparison with a brass tube.

\section{CONCLUSIONS}

A practical method to evaluate the transverse impedance of accelerator components at low frequencies has been reported. A modification of the classical two wire method which is running into sensitivity problems below a few $\mathrm{MHz}$ has been successfully tested and compared with theoretical expectations. This modified method, which uses essentially a loop with a certain number of turns, is better matched to the transverse impedance to be evaluated (at least in some practical cases of interest for LHC) than the normal two wire technique. Also it can be used from a few $\mathrm{Hz}$ onwards while the upper operational frequency is limited to the first loop resonance. To asses the validity of the method, measurements on a lossy circular beam pipe have been checked against theoretical results.

\section{ACKNOWLEDGMENTS}

The authors are grateful to H. Hahn (BNL) for interesting and stimulating discussions.

\section{REFERENCES}

[1] G. Nassibian, F. Sacherer, CERN, PS-BR 77-40, 1977.

[2] A.W. Chao, M. Tinger (Eds.), Handbook of Accelerator Physics and Engineering (World Scientific, 1999).

[3] B. Zotter, A. Kheifets, Impedance and Wakes in High-Energy Particle Accelerators (World Scientific, 1998).

[4] L. Vos, CERN-AB-2003-005 ABP, 2003.

[5] A. Burov, V. Lebedev, Proc. of EPAC 2002, Paris, France, pp. 1452 (2002).

[6] V. Lebedev, Multi-Bunch Instabilities in VLHC, presented in VLHC Workshop, SLAC, March 2001. 